\title{
Álgebra abstracta: de grupos a preliminares de la Teoría de Galois
}

Pérez Terrazas, J. E. y Guerrero Lara, E. Álgebra abstracta: de grupos a preliminares de la Teoría de Galois. Mérida, Yucatán: Editorial de la Universidad Autónoma de Yucatán, 2010, 132 pp., ISBN 978-607-7573-43-2.

\section{Jorge Eduardo Macías Díaz ${ }^{1 *}$}

Macías Díaz, J. E. Álgebra abstracta: de grupos a preliminares de la Teoría de Galois. Reseña. Investigación y Ciencia de la Universidad Autónoma de Aguascalientes. Número 69: 98-99 septiembre-diciembre 2016.

Álgebra abstracta: de grupos a preliminares de la Teoría de Galois es un tratado de álgebra moderna que abarca desde el mismo preámbulo de las estructuras algebraicas (monoides, semigrupos) hasta las teorías de grupos y anillos, las extensiones de campos y la Teoría de Galois. Los temas propuestos pueden ser dictados holgadamente en un par de semestres, lo que da una oportunidad interesante para resolver ejercicios dentro del aula de clase. Por su temario, por la seriación de los contenidos y el nivel de profundidad de los mismos, este libro es un auxiliar idóneo para cursos introductorios de álgebra abstracta en cualquier licenciatura en matemáticas del país.

En este libro de texto, los autores han propuesto listas de ejercicios para ser resueltos en el salón de clase. Desde mi punto de vista, el contar con estos problemas abre muchas oportunidades para implementar estrategias de aprendizaje en el aula e, incluso, de autoaprendizaje. Los ejercicios varían en su complejidad (desde aquellos que se resuelven inmediatamente aplicando definiciones y teoremas, hasta los que requieren de cierto ingenio y madurez matemática). Afortunadamente, aquellos que no son lo suficientemente obvios cuentan con un bosquejo de solución.

Otra cualidad del libro es la extensa lista de ejercicios propuestos que aparecen en cada sección. Nuevamente, los ejercicios varían en su complejidad, pero esta variabilidad da pie a la posibilidad de que los estudiantes refuercen la

\footnotetext{
Departamento de Matemáticas y Física, Centro de Ciencias Básicas, Universidad Autónoma de Aguascalientes.

* Autor para correspondencia: jemacias@correo.uaa.mx
}

comprensión de definiciones y resultados, y que desarrollen agudeza matemática en la resolución de problemas algebraicos. Algunos de dichos problemas consisten en establecer afirmaciones que se postularon sin demostración en el texto; de tal manera, estos ejercicios dan continuidad al libro en su conjunto. Otros problemas son empleados ulteriormente en el desarrollo del curso. Claramente, esta práctica muestra al estudiante el valor

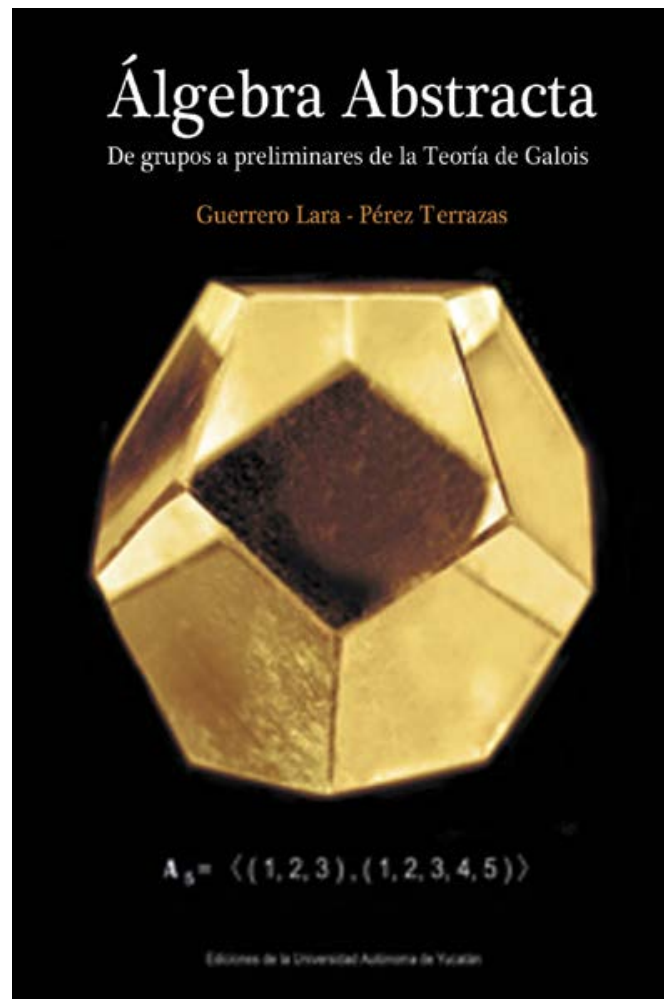

Portada del libro Álgebra abstracta: de grupos a preliminares de la Teoría de Galois. 
pragmático de resolver los ejercicios propuestos, e impulsa al lector a tratar de demostrarlos.

A la luz de este libro, la presentación del álgebra es amena y atractiva. En efecto, la clasificación rigurosa de párrafos en 'definiciones', 'lemas', 'teoremas', 'corolarios', 'ejemplos', 'observaciones', 'demostración', etcétera, proporciona al estudiante la conveniencia de asignar un sentido utilitario a cada porción de texto. Sin embargo, este formalismo matemático nunca se torna tedioso o aburrido. Ello se debe a que los autores parecieran llevar al lector de la mano en su discurso. De hecho, pareciese que son los autores mismos quienes saltan de las páginas $y$, con lujo de argumentos elocuentes, muestran la belleza del rigor propio de las verdaderas matemáticas. Como ejemplo, podemos citar los párrafos que preceden al teorema de Cayley, en los cuales los autores proporcionan la demostración del teorema sin mencionarlo abiertamente al lector.

Por otra parte, con el fin de determinar más objetivamente las bondades de este libro, es conveniente compararle con textos clásicos del área. Para ello, habremos de referirnos a algunos de los libros que se citan en la bibliografía (la cual, quepa mencionar, es vasta y completa). Por ejemplo, comparado con los libros clásicos de Fraleigh, de Herstein, de Hungerford, de Jacobson o de Rotman, Álgebra abstracta: de grupos a preliminares de la Teoría de Galois es un tratado más moderno en cuanto a su desarrollo temático, a su notación y exposición. En particular, Herstein, Jacobson y Fraleigh adolescen de una exposición concisa a la luz del formalismo de las matemáticas actuales. Desafortunadamente, muchos de los libros tradicionales fueron textos extraordinarios hasta finales del siglo pasado. Sin embargo, en la alborada del siglo XXI se han ido tornando obsoletos.

Otra de las virtudes de Álgebra abstracta: de grupos a preliminares de la Teoría de Galois es que ha sido escrito pensando en estudiantes de licenciatura. Los libros arriba mencionados, particularmente el Hungerford, el Jacobson y el Rotman, fueron diseñados teniendo en mente a estudiantes de posgrado en matemáticas puras. Si bien dichos libros brindan tratados más profundos en algunos temas, también es cierto que requieren cierta formación matemática en el lector más allá del nivel de licenciatura. No olvidemos, por ejemplo, que Hungerford no demuestra sus teoremas a completamente: sólo proporciona bosquejos de demostraciones para que el lector escriba los detalles restantes.

En resumen, este lector ha encontrado en el libro Álgebra abstracta: de grupos a preliminares de la Teoría de Galois una obra que merece ser difundida entre las carreras de licenciatura del país, y empleada como libro de texto en cursos de álgebra abstracta. 